\title{
Numerical analysis of heat transfer in cooling of fish packages
}

\begin{abstract}
The present work aims at finding the optimal finite difference scheme for the solution of problems involving pure heat transfer from the surface of solids suddenly exposed to a cooling environment. Fish samples in the form of infinite slab were considered, and a generalized mathematical model was constructed in dimensionless form. A more representative and accurate set of experimental data is chosen from the experimental work for comparison with the numerical results and evaluation of numerical schemes. In the analysis, a fully explicit finite difference scheme, an implicit finite difference scheme and different combination schemes with varying values of weighting factor are thoroughly studied. The characteristic dimension (half thickness of the slab) is divided into a number of divisions; $\mathrm{n}=$ 5, 10, 20, 30, 40, 50 and 100, respectively. All the possible options of the Fourier number increments are taken one by one to give the best convergence and minimal truncation error. The simplest explicit finite difference scheme with $\mathrm{n}=10$ and Fourier number increments one sixth of the square of the space division size appears to be highly reliable and accurate for such applications.
\end{abstract}

Keyword: Heat transfer, Cooling, Fish package, Experiment, Numerical study 